\title{
Influência da geometria e da temperatura na cinética de secagem de tomate (Lycopersicum esculentum)
}

\author{
Influence of temperature and geometry in the drying kineticof tomato (Lycopersicum esculentum)
}

\author{
Eliana Janet SANJINEZ-ARGANDOÑA ${ }^{1 *}$, Ivanise Guilherme BRANCO ${ }^{2}$, \\ Tiemi Umebara BITTENCOURT ${ }^{3}$, Cláudia Leite MUNHOZ ${ }^{1}$
}

\begin{abstract}
Resumo
O tomate é um fruto muito perecível por causa do seu conteúdo de umidade. A secagem é uma das práticas industriais mais utilizadas em alimentos para manter a qualidade do produto final. A pesquisa foi desenvolvida para estudo dos parâmetros de secagem de tomate (Lycopersicon esculentum Mill), cv Carmen, com relação ao tipo de corte (meio e um quarto) e à temperatura de processo (60 e $\left.70{ }^{\circ} \mathrm{C}\right)$, bem como à escolha do tempo de secagem para a obtenção de um produto com umidade de $45 \%$ (base úmida). As cinéticas de secagem foram determinadas experimentalmente por convecção forçada e ajustadas ao modelo de Page. Os resultados obtidos mostraram que a geometria de corte influenciou na taxa de secagem e no tempo de desidratação. Os tomates cortados em quatro partes e desidratados a $70{ }^{\circ} \mathrm{C}$ alcançaram umidade de $45 \%$ em menor tempo (10 horas), quando comparados aos tomates com o corte em metades. O modelo de Page forneceu bom ajuste nas cinéticas de secagem.

Palavras-chave: tomate; geometria de corte; cinética de secagem; modelo de Page.
\end{abstract}

\begin{abstract}
Tomato is a very perishable fruit due to its content of moisture. Drying is one of the most used industry practices in foods to keep the final product quality. The aim of this research was to study the parameters of the drying of tomatoes (Lycopersicon esculentum Mill), cv Carmen in terms of the type of cut $(1 / 2$ and $1 / 4)$ and the process temperature $\left(60\right.$ and $\left.70{ }^{\circ} \mathrm{C}\right)$, as well as the choice of the setting time for the attainment of a product with $45 \%$ moisture. The kinetics of drying data were determined experimentally by forced convection and adjusted to the Page model. The results showed that the cutting geometry influenced the rate of drying and time of dehydration. The tomatoes cut into four pieces and dehydrated at $70{ }^{\circ} \mathrm{C}$ reached $45 \%$ humidity sooner (10 hours) than those cut in halves. The Page model provided good fit to the drying kinetics data.

Keywords: tomato; cutting geometry; drying kinetics; Page model.
\end{abstract}

\section{Introdução}

O tomate é uma das culturas nacionais de maior importância econômica, pois é a hortaliça mais industrializada na forma de inúmeros subprodutos, como extrato, polpa, pasta e, mais recentemente, o tomate seco, cujo mercado vem crescendo consideravelmente. Atualmente, o Brasil é um dos maiores produtores mundiais, junto com Estados Unidos, Itália, Turquia, Espanha, Egito e México. Segundo o Instituto Brasileiro de Geografia e Estatística (IBGE, 2007), sua área cultivada foi de 57 mil ha, com uma produção de 3.4 mil toneladas, apresentando um rendimento de $59.2 \mathrm{~kg} \cdot \mathrm{ha}^{-1}$, sendo os Estados de São Paulo, Goiás e Minas Gerais os maiores produtores brasileiros, responsáveis por $57,5 \%$ da produção nacional e com 57,3\% de área plantada. Apresenta, porém, perdas pós-colheita na ordem de 25 a 50\% (Pacco, 2008), em consequência de sua alta perecibilidade, cultivar utilizada, modo de beneficiamento, local, época do ano, classe do produto, etapa da cadeia considerada e outros fatores (HENZ; MORETTI, 2005).
O maior consumo de tomates é na forma in natura em saladas e industrializados, como extrato de polpa concentrado, polpa, pedaços de tomate com adição de especiarias. Porém, com as mudanças dos hábitos alimentares dos consumidores, os tomates secos têm aumentado sua popularidade e consumo (AKANBI; ADEYEMI; OJO et al., 2006).

Segundo Murari (2001), o tomate seco é um produto diferenciado tanto no aspecto do processamento como na comercialização. Trata-se de tomates não inteiros desidratados até umidade intermediária, mantendo sua textura macia. O tomate seco é comercializado imerso em óleo vegetal e temperado com sal, alho, orégano e outros condimentos. No Brasil, por tratar-se de um produto relativamente novo, o processamento é feito por pequenas e microempresas, em geral, próximas às regiões produtoras. Os métodos utilizados são bastante artesanais, pelos quais a preparação do tomate é

${ }^{1}$ Universidade Federal da Grande Dourados - UFGD, Rod. Dourados-Itahum,Km 12, Dourados - MS, Brasil, E-mail: eliana.argandona@ufgd.edu.br; clmunhoz@yahoo.com

2 Universidade Estadual Paulista - UNESP, Campus de Assis - SP, Brasil, E-mail: ivanisebranco@bol.com.br

3 Universidade Federal do Paraná - UFPR, E-mail: tittuca@hotmail.com

${ }^{*}$ Corresponding author 
manual e a secagem realizada ao sol ou em fornos convencionais. O tomate seco no Brasil é consumido em forma de aperitivo, guarnição, pizzas e como ingrediente de receitas e formulações alimentícias.

No entanto, são poucas as referências disponíveis sobre a variedade do fruto mais recomendada e os parâmetros de secagem, como temperatura adequada, teor de umidade final, dentre outros (CAMARGO, 2000). Os tomates secos encontrados no mercado apresentam variabilidade significativa quanto às características de textura e cor. Esses parâmetros estão diretamente relacionados com a temperatura e o tempo de secagem, influenciando na aceitabilidade do produto pelo consumidor (BORDERÍAS; MONTERO, 1988). Assim, o estudo de parâmetros de processo de secagem associado às formulações de molhos para conserva dos produtos é de interesse científico e industrial.

A modelagem de processos de secagem é fundamental para a seleção e a otimização dos mesmos. Entretanto, as equações de transferência de calor e massa demandam considerável conhecimento de cálculo numérico para sua solução analítica (SIMAL; DEYÁ; ROSELLÓ, 1997; SIMAL et al., 2005; DOYMAZ, 2004). Assim, o modelo de Page (Equação 1) tem sido amplamente usado para simular as curvas de secagem de pêras (PARK; BIN; BROD, 2002), mamão (EL-AOUAR et al., 2003), cenoura (DOYMAZ, 2004), kiwi (SIMAL et al., 2005), entre outros.

$\frac{X_{t}-X_{\infty}}{X_{o}-X_{\infty}}=\exp \left(-k t^{n}\right)$

Na qual: $X_{o}, X_{t}$ e $X_{\infty}=$ umidades inicial, no tempo $t$ e no equilíbrio, respectivamente $\left(\mathrm{kg}_{\mathrm{H}_{2} \mathrm{O}} / \mathrm{kg}_{\mathrm{ms}}\right) ; \mathrm{k} \mathrm{e} \mathrm{n}=$ constantes do modelo; $\mathrm{t}=$ tempo de secagem (segundos).

Portanto, os objetivos do trabalho foram estudar a influência do tipo de corte e da temperatura no processo de desidratação do tomate, bem como determinar os parâmetros de secagem para obter um produto desidratado com $45 \%$ de umidade no menor tempo possível.

\section{Material e métodos}

\subsection{Matéria-prima}

Vinte quilos de tomates (Lycopersicon esculentum Mill) de mesa, $c v$ Carmen, foram adquiridos no mercado local de Guarapuava-PR.

\subsection{Métodos}

\section{Preparo das amostras}

Os frutos foram acondicionados em caixas e mantidos à temperatura ambiente $\left(24 \pm 2{ }^{\circ} \mathrm{C}\right)$ até que apresentassem a cor adequada para realizar os experimentos, definida pela cor vermelha seguindo o padrão de cores disponibilizado na carta de comercialização do Programa Horti \& Fruti de produtos hortigranjeiros (CEAGESP, 2005).

Os tomates para processamento foram selecionados de acordo com a aparência (maturação, tamanho e cor), lavados e sanitizados com solução de cloro ativo (200 ppm) e secos em papel absorvente. Logo a seguir, foram submetidos a dois tipos de cortes: em metades (1/2) e em quatros partes (1/4), em relação ao eixo maior do fruto; foram retiradas as sementes, lavados em água destilada, secos novamente com papel absorvente, pesados e distribuídos em bandejas.

\section{Processo de secagem}

A desidratação dos tomates foi realizada nas temperaturas de 60 e $70^{\circ} \mathrm{C}$, para cada tipo de corte, em um secador de bandejas com velocidade de ar quente a $0,5 \mathrm{~m} /$ segundo, verificada pelo uso de um anemômetro.

As curvas de secagem foram obtidas por pesagens periódicas dos tomates e a umidade determinada por método gravimétrico. As pesagens foram realizadas em intervalos de 15 minutos durante a primeira hora de secagem, de 30 minutos nas 10 horas seguintes e de 2 horas até o equilíbrio dinâmico entre as amostras. Foram construídas cinéticas de secagem do tomate até o equilíbrio nos diferentes tratamentos com a finalidade de conhecer o tempo ideal para se obter um produto com umidade final de $45 \%$.

As curvas de secagem foram construídas com umidade em base seca ( $\mathrm{kg}$ de água. $\mathrm{kg}^{-1}$ sólido seco) em relação ao tempo. A taxa de secagem $(\mathrm{dX} / \mathrm{dt})$ foi calculada pela derivada da umidade em relação ao tempo (Equação 2). Posteriormente, as cinéticas de secagem foram ajustadas pela equação de Page (Equação 1), para possibilitar a determinação do tempo ideal de secagem dos tomates para conserva.

$\frac{d X}{d t}=\frac{X_{n-1}-X_{n}}{t_{n-1}-t_{n}}$

Na qual: $\mathrm{t}=$ tempo (minutos); $\mathrm{n}=$ número de leituras; $\mathrm{dX} / \mathrm{dt}=$ taxa de secagem $\left(\mathrm{kg}_{\mathrm{H} 2 \mathrm{O}} / \mathrm{kg}_{\mathrm{ms} \cdot \min }\right) ; \mathrm{X}_{\mathrm{n}}$ e $\mathrm{X}_{\mathrm{n}-1}=$ umidade $\left(\mathrm{kg}_{\mathrm{H} 2 \mathrm{O}} / \mathrm{kg}_{\mathrm{ms}}\right)$ no tempo $n$ e no tempo $n-1$.

Para validação do modelo, foram calculados o coeficiente de determinação $\left(\mathrm{R}^{2}\right)$ e o erro médio relativo $(\mathrm{E})$, que é definido como a diferença relativa entre os valores experimentais $\mathrm{e}$ preditos (Equação 3), sendo considerado preditivo o modelo que apresentar valores de $\mathbf{E}$ menores que $10 \%$.

$$
E(\%)=\frac{100}{N} \Sigma \frac{\left|M_{\exp }-M_{p r e d}\right|}{M_{\exp }}
$$

Na qual: $M_{\text {exp }}$ e $M_{\text {pred }}=$ valores obtidos experimentalmente e os preditos pelo modelo; $\mathrm{N}=$ número de pontos experimentais.

O cálculo desses parâmetros estatísticos foi realizado por meio dos pacotes Statistica v. 5.0 e Origin v. 5.0.

\section{Resultados e discussão}

\subsection{Efeito da temperatura e da geometria de corte na secagem de tomates}

A Figura 1 mostra as cinéticas de secagem a 60 e $70{ }^{\circ} \mathrm{C}$ de tomates cortados em metades e em quatro partes, em 
função da umidade em base seca e do tempo. Percebe-se a influência da temperatura nas cinéticas, uma vez que maiores valores deste parâmetro resultam na diminuição da umidade e, consequentemente, na redução do tempo de secagem. As amostras de tomate na geometria de um quarto e temperaturas de secagem de 60 e $70^{\circ} \mathrm{C}$ apresentaram comportamento cinético bastante similar, alcançando o equilíbrio após 16,5 horas de processo.

$\mathrm{Na}$ geometria de corte em metades o comportamento foi diferente nas temperaturas estudadas. Em $60{ }^{\circ} \mathrm{C}$, houve uma rápida diminuição da umidade nos primeiros quinze minutos, sendo que esse comportamento não foi observado a $70^{\circ} \mathrm{C}$. É possível que tenha ocorrido um período de repouso antes de essas amostras terem sido colocadas no secador, ocorrendo assim transferência de água do interior para a periferia do fruto, o que aumentou a disponibilidade de água superficial a ser evaporada. O equilíbrio alcançado nas amostras desidratadas a $60{ }^{\circ} \mathrm{C}$ foi em 26,7 horas de secagem e, a $70{ }^{\circ} \mathrm{C}$, em 20,5 horas, aproximadamente. A umidade de equilíbrio para os tomates desidratados a $60{ }^{\circ} \mathrm{C}$ foi de aproximadamente $0,35 \mathrm{~kg}$ de água. $\mathrm{kg}^{-1}$ matéria seca e, na temperatura de $70^{\circ} \mathrm{C}$, foi de $0,23 \mathrm{~kg}$ de água. $\mathrm{kg}^{-1}$ matéria seca.

A partir do exposto, verifica-se, além da temperatura, a influência do tipo de corte na secagem. O corte em quatro partes favoreceu a evaporação d'água devido à maior área de contato, diminuindo o tempo de processo para alcançar o equilíbrio.

Para a obtenção de um produto com $45 \%$ de umidade final, o tempo de secagem a $60{ }^{\circ} \mathrm{C}$ para amostras em metades foi de 21,4 horas e, para amostras cortadas em quatro partes, foi de 12,5 horas. A $70^{\circ} \mathrm{C}$, para amostras em metades e em quatro partes, a umidade prevista foi alcançada em 16,5 horas e 10 horas, respectivamente.

O comportamento de secagem pode apresentar taxas de secagem constante e/ou decrescente. Para produtos biológicos, o comportamento de secagem é, geralmente, decrescente. Esse comportamento é determinado pela migração interna de umidade (PARK; BIN; BROD, 2002; BENDLIN, 2003). $\mathrm{Na}$ Figura 2, observa-se, no geral, comportamento de taxa de secagem decrescente. Isto demonstra que a difusão é provavelmente o mecanismo físico que governa o movimento da água no fruto. A evaporação da água dentro do alimento é influenciada por diversos fenômenos, como: capilaridade, ligação físico-química da água às substâncias sólidas presentes no alimento, migração de solutos, deformação do produto, entre outros; note-se que tais fenômenos oferecem grande resistência (BENDLIN, 2003). Pena et al. (2008), na secagem da fibra residual do maracujá, e Azoubel et al. (2009), na secagem de caju com e sem pré-tratamento osmótico, observaram também taxas de secagem decrescentes.

Para melhor visualização do comportamento da desidratação, as taxas de secagem foram calculadas em função do conteúdo de umidade (Figura 2).

A área de contato da amostra com o fluxo de ar quente é fator determinante da velocidade do processo de secagem. Assim, a secagem de tomate em quatro partes foi o processo que apresentou maior taxa de secagem nas duas temperaturas.
Similar comportamento foi obtido por Camargo (2000), na secagem do tomate das cultivares Débora Plus e Rio Grande.

\subsection{Ajuste matemático das cinéticas de secagem}

As Figuras 3 a 6 mostram o ajuste dos dados experimentais pelo modelo de Page para as amostras de tomate com geometria de corte de meio e um quarto, desidratadas a 60 e $70^{\circ} \mathrm{C}$. Os parâmetros do modelo são apresentados na Tabela 1 .

Os parâmetros obtidos para o modelo de Page proporcionaram baixos valores de porcentagem de erro relativo médio entre os dados de umidade experimentais e os estimados (Tabela 1). Segundo Karathanos e Belessiotis (1999), o valor de $\mathrm{n}$ aumenta quando a fruta é desidratada com casca ou pele;

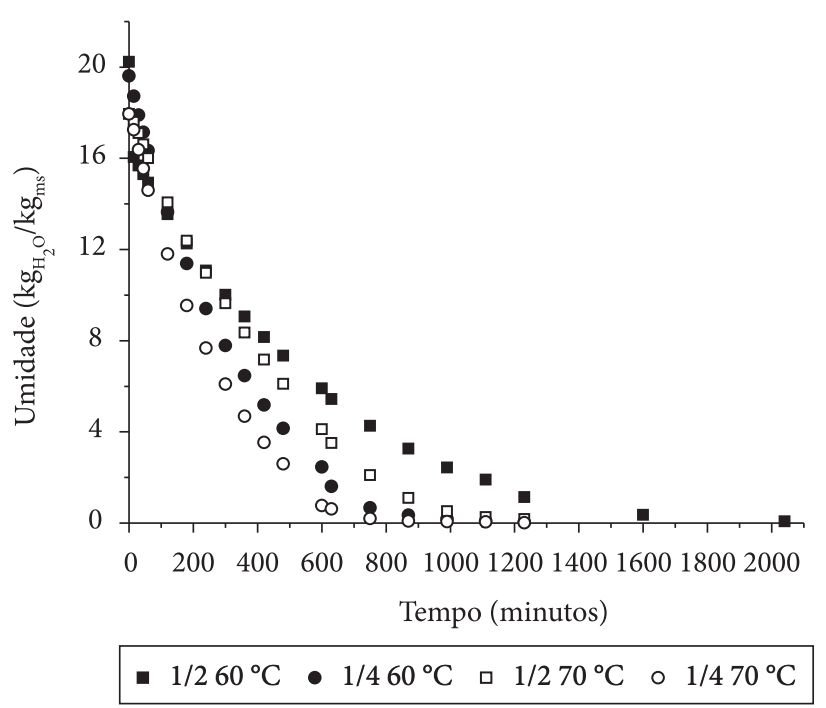

Figura 1. Curvas de secagem do tomate em metades a $60{ }^{\circ} \mathrm{C}(\boldsymbol{\square})$ e $70{ }^{\circ} \mathrm{C}(\square)$ e em quatro partes a $60{ }^{\circ} \mathrm{C}(\bullet)$ e $70{ }^{\circ} \mathrm{C}(\circ)$.

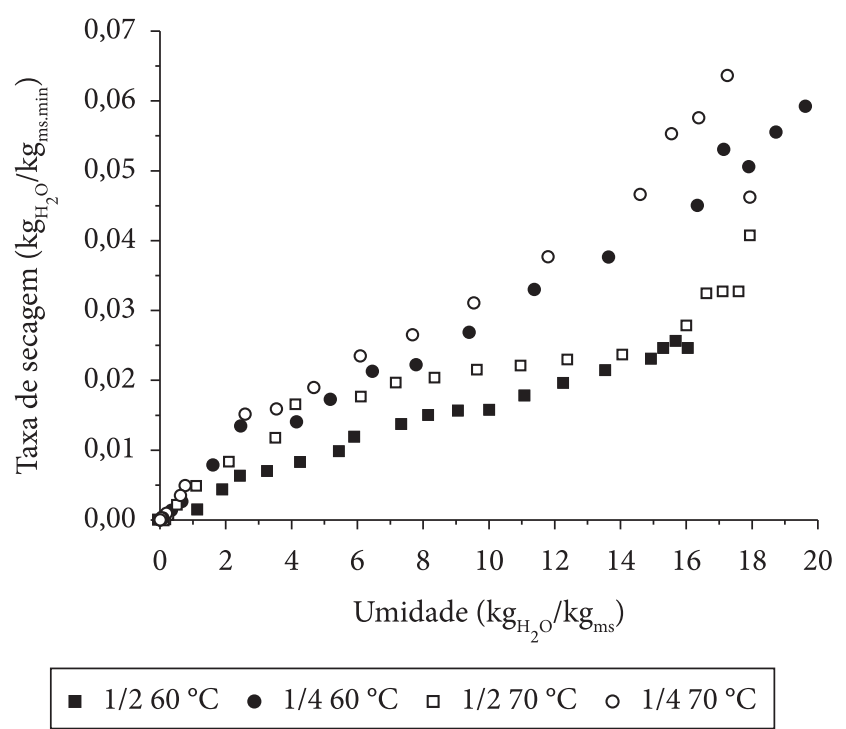

Figura 2. Taxa de secagem do tomate em metades a $60{ }^{\circ} \mathrm{C}(\boldsymbol{\square})$ e $70{ }^{\circ} \mathrm{C}(\square)$ e em quatro partes a $60{ }^{\circ} \mathrm{C}(\bullet)$ e $70{ }^{\circ} \mathrm{C}(\circ)$. 
os autores observaram ainda que quanto maior a espessura da casca, os valores de $\mathrm{n}$ foram mais elevados, variando de 1,02 a 1,79 . Com exceção dos valores obtidos para os tomates em metades desidratados a $60{ }^{\circ} \mathrm{C}$, os valores para o parâmetro n variaram de 1,11 a 1,23 , os quais se encontram dentro do intervalo obtido pelos autores. $\mathrm{Na}$ desidratação de fatias de

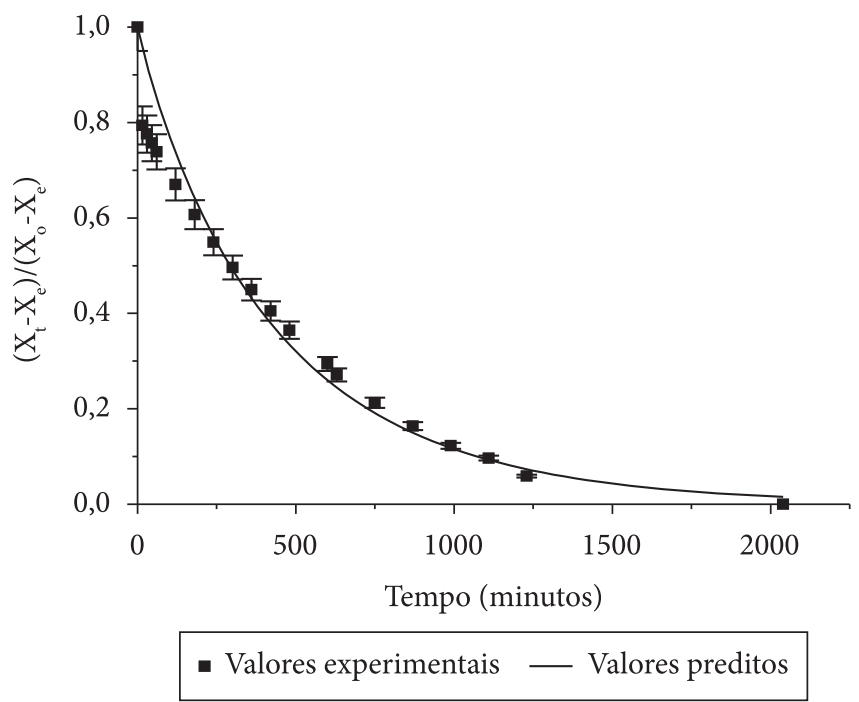

Figura 3. Modelo de Page para o tomate desidratado a $60{ }^{\circ} \mathrm{C} \mathrm{em}$ metades. Valores experimentais ( $\mathbf{a})$ e valores preditos (linha contínua).

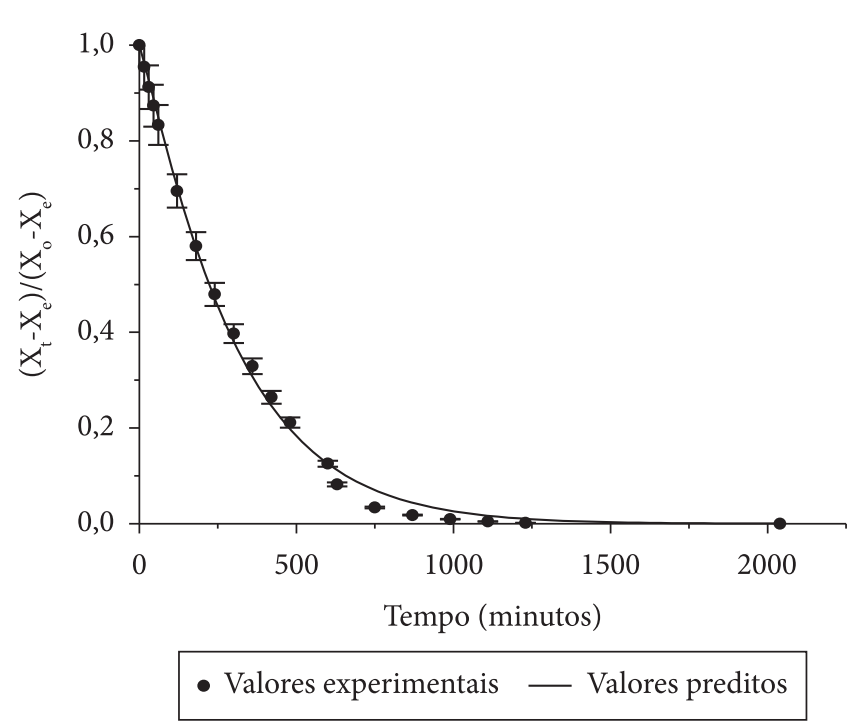

Figura 4. Modelo de Page para o tomate desidratado a $60^{\circ} \mathrm{C}$ em quatro partes. Valores experimentais $(\bullet)$ e valores preditos (linha contínua). kiwis sem casca, Simal et al. (2005) obtiveram valores de n de 0,796, independentemente da temperatura de secagem e da geometria do corte. Resultados similares foram encontrados por Azzouz et al. (2002) para a secagem de uvas, que concluíram que n é função da velocidade do ar de secagem e da umidade inicial do produto, enquanto que o parâmetro k é função desta e da

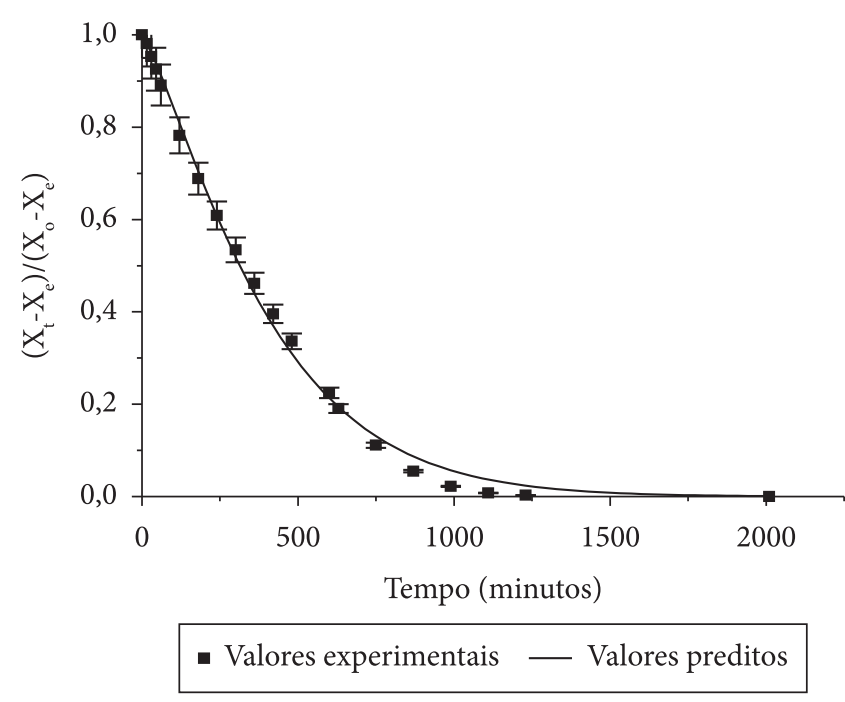

Figura 5. Modelo de Page para o tomate desidratado a $70{ }^{\circ} \mathrm{C} \mathrm{em}$ metades. Valores experimentais ( $\mathbf{\square})$ e valores preditos (linha contínua).

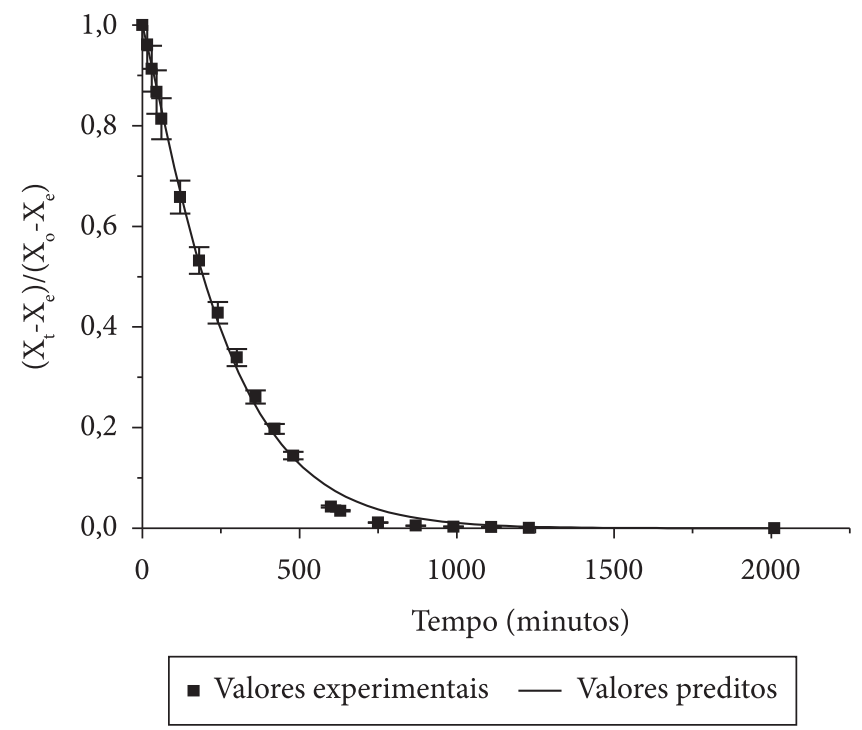

Figura 6. Modelo de Page para o tomate desidratado a $70^{\circ} \mathrm{C}$ em quatro partes. Valores experimentais ( $\mathbf{\square})$ e valores preditos (linha contínua).

Tabela 1. Valores dos parâmetros do modelo de Page para o tomate cortado em metade e um quarto, e desidratado a 60 e $70{ }^{\circ} \mathrm{C}$.

\begin{tabular}{lcccc}
\hline \multicolumn{1}{c}{ Amostra } & \multicolumn{3}{c}{ Modelo de Page } \\
\cline { 2 - 5 } & $\mathrm{k}\left(\right.$ minuto $\left.^{-1}\right)$ & $\mathrm{n}$ & $\mathrm{R}^{2}$ & $\mathrm{E}(\%)$ \\
\hline${\text { Corte de } 1 / 2 \text { a } 60^{\circ} \mathrm{C}}_{\text {Corte de } 1 / 4 \text { a } 60^{\circ} \mathrm{C}}$ & 0,01721 & 0,6721 & 0,971 & 3,83 \\
Corte de $1 / 2$ a $70^{\circ} \mathrm{C}$ & 0,00172 & 1,1085 & 0,998 & 1,45 \\
Corte de $1 / 4$ a $70^{\circ} \mathrm{C}$ & 0,00057 & 1,2344 & 0,998 & 1,76 \\
\hline
\end{tabular}


temperatura de secagem e do conteúdo inicial de umidade. Neste estudo, não houve influência da umidade sobre o parâmetro $\mathrm{k}$. Waughon e Pena (2008) obtiveram excelentes precisões com o uso do modelo de Page na predição dos dados de secagem da fibra residual do abacaxi em camada delgada. Doymaz (2007) também utilizou o modelo de Page para desidratação de tomates, apresentando um bom ajuste aos dados experimentais de secagem a $55-70{ }^{\circ} \mathrm{C}$, sendo encontrado $\mathrm{n}$ de 1,1876 e $\mathrm{k}$ de 0,0006 para a temperatura de $70{ }^{\circ} \mathrm{C}$.

\section{Conclusões}

A geometria de corte apresentou maior influencia na taxa de secagem do que a temperatura de processo.

Os tomates cortados em quatro partes desidratados a $70{ }^{\circ} \mathrm{C}$ com velocidade de ar quente a $0,5 \mathrm{~m} /$ segundo alcançaram a umidade de $45 \%$ em menor tempo (10 horas de secagem).

O modelo matemático de Page forneceu um bom ajuste para os tomates cortados em ambas as geometrias de corte.

\section{Referências bibliográficas}

AKANBI, C. T.; ADEYEMI, R. S.; OJO, A. Drying characteristics and sorption isotherm of tomato slices. Journal of Food engineering, v. 73, n. 2, p. 157-163, 2006. http://dx.doi.org/10.1016/j. jfoodeng.2005.01.015

AZOUBEL, P. M. et al. Effect of osmotic dehydration on the drying kinetics and quality of cashew Apple. International Journal of Food Science and Technology, v. 44, p. 980-986, 2009. http://dx.doi. org/10.1111/j.1365-2621.2008.01783.x

AZZOUZ, S. et al. Moisture diffusivity and drying kinetic equation of convective drying of grapes. Journal of Food Engineering, v. 55, p. 323-330, 2002. http://dx.doi.org/10.1016/S0260-8774(02)00109-7

BENDLIN, R. C. S. Secagem convectiva de erva-mate (Ilex paraguariensis). 2003. $77 \mathrm{f}$. Dissertação (Mestrado em Engenharia de Alimentos)-Departamento de Engenharia Química e de Alimentos, Universidade Federal de Santa Catarina, Florianópolis, 2003.

BORDERÍAS, A. J.; MONTERO, P. Fundamentos de la funcionalidad de las proteínas en alimentos. Revista Agroquímica y Tecnologia de Alimentos, v. 28, n. 2, p. 159-169, 1988.

CAMARGO, G.A. Secagem de tomate (Lycopersicon esculentum Mill): Estudo de parâmetros com base na qualidade final. 2000. 72 f. Dissertação (Mestrado em Engenharia Agrícola)-Faculdade de Engenharia Agrícola, Universidade Estadual de Campinas, Campinas, 2000

COMPANHIA DE ENTREPOSTOS E ARMAZÉNS GERAIS DE SÃO PAULO - CEAGESP. Programa Horti \& Fruti Padrão-
Classificação do Tomate. Disponível em: <www.cati.sp.gov.br/ novacati/index.php>. Acesso em: 12 abr. 2005.

DOYMAZ, I. Convective air drying characteristics of thin layer carrots. Journal of Food Engineering, v. 61, n. 1, p. 359-364, 2004. http:// dx.doi.org/10.1016/S0260-8774(03)00142-0

DOYMAZ, I. Air-drying characteristics of tomatoes. Journal of Food Engineering, v. 78, n. 4, p. 1291-1297, 2007. http://dx.doi. org/10.1016/j.jfoodeng.2005.12.047

EL-AOUAR, A.A. et al. Drying kinetics of fresh and osmotically pretreated papaya (Carica papaya L.) Journal of Food Engineering, v. 59, p. 85-91, 2003. http://dx.doi.org/10.1016/S0260-8774(02)00434-X

HENZ, G. P.; MORETTI, C. L. Tomate: Manejo Pós Colheita, 2005. Disponível em: <http://www.grupocultivar.com.br/arquivos/ hf30_manejotomate.pdf>. Acesso em: 28 maio 2009.

INSTITUTO BRASILEIRO DE GEOGRAFIA E ESTATÍSTICA - IBGE Banco de Dados Agregados: Agricultura. Quantidade Produzida, 2007. Disponível em: http://www.sidra.ibge.gov.br/bda/agric/ default.asp? $\mathrm{t}=2 \& \mathrm{z}=\mathrm{t} \& \mathrm{o}=11 \& \mathrm{u} 1=1 \& \mathrm{u} 2=1 \& \mathrm{u} 3=1 \& \mathrm{u} 4=1 \& \mathrm{u} 5=1$ \&u6=1 Acesso em: fev. 2008.

KARATHANOS, V. T.; BELESSIOTIS, V. G. Application of a thin-layer equation to drying data of fresh and semi-dried fruits. Journal of Agricultural Engineering Research, v. 74, n. 4, p. 355-361, 1999. http://dx.doi.org/10.1006/jaer.1999.0473

MURARI, R. C. D. L. Cinética da desidratação osmo-convectiva de tomates. 2001. Dissertação (Mestrado em Engenharia de Alimentos)-Instituto de Biociências, Letras e Ciências Exatas, Universidade Estadual Paulista, São José do Rio Preto, 2001.

PACCO, H. C. Desenvolvimento de um sistema de bomba de calor água/água para resfriamento e secagem de tomates. Tese (Doutorado em Engenharia Agrícola)-Faculdade de Engenharia Agrícola, Universidade Estadual de Campinas, Campinas, 2008.

PARK, K.J.; BIN, A.; BROD, F. P. R. Drying of pear d'Anjou with and without osmoticdehydration. Journal of Food Engineering, v. 56, p. 97-103, 2002. http://dx.doi.org/10.1016/S0260-8774(02)00152-8

PENA, R. S. et al. Estudo da secagem da fibra residual do maracujá. Revista Brasileira de Tecnologia Agroindustrial, v. 2, n. 1, p. 1-13, 2008.

SIMAL, S.; DEYÁ, E.; ROSELLÓ, C. Simple modelling of drying curves of fresh and osmotically pre-dehydrated apple cubes. Journal of Food Engineering, v. 33, p. 139-150, 1997. http://dx.doi. org/10.1016/S0260-8774(97)00049-6

SIMAL, S. et al. Use of exponential, Page's and diffusional models to simulate the drying kinetics of kiwi fruit. Journal of Food Engineering, v. 43, p. 109-114, 2005. http://dx.doi.org/10.1016/ S0260-8774(99)00139-9

WAUGHON, T. G. M.; PENA, R. S. Modelagem da secagem em camada delgada da fibra residual do abacaxi. Boletim do CEPPA, v. 26, n. 2, p. 297-306, 2008. 